\title{
Pneumonia associated with a single intratracheal dose of bleomycin in mice
}

\author{
Leila Safaeian, Pharm.D. ${ }^{1}$, Noushin Afshar Moghaddam, $P h D^{2}$ \& Abbas Jafarian, $P h D^{3}$
}

Bleomycin-induced pulmonary fibrosis in mice is a commonly used animal model for human lung fibrosis ${ }^{1}$. For a study of bleomycin-induced pulmonary fibrosis, we obtained 30 female NMRI mice (25-30 g) from the Pasteur Institute (Tehran, Iran). Animals were housed in standard rodent cages in a mouse room on a 12-hr light/ dark cycle. The mice had ad libitum access to water and laboratory rodent chow. Experiments were carried out according to the International Guiding Principles for Biomedical Research Involving Animals as developed by the Council for International Organizations of Medical Sciences (CIOMS).

To induce pulmonary fibrosis, we administered a single intratracheal dose of bleomycin to each mouse according to the protocol developed by Schrier et al. ${ }^{2}$. Under ketamine anesthesia $(75 \mathrm{mg} / \mathrm{kg}$ i.p.), doses of $0.075 \mathrm{U}$ bleomycin in $50 \mu \mathrm{l}$ of sterile isotonic saline were instilled in mice intratracheally. Control mice were given the same volume of sterile saline. Six animals were used in each control and experimental group.

Each week for four weeks after bleomycin instillation, we euthanized a group of 6 mice by ketamine overdose, removed the lungs, and perfused the left lung lobe with $10 \%$ neutral buffered formalin (NBF) solution via the trachea. The perfused lungs were then fixed in a $10 \%$ NBF solution before processing for histological examination. After sectioning and histological processing, sections

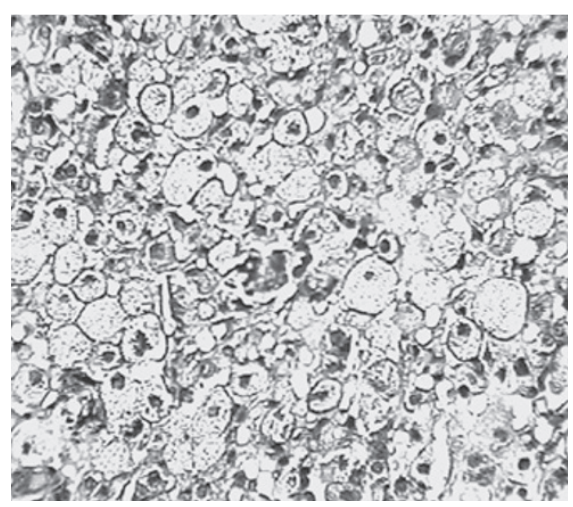

FIGURE 1 | Black and white photomicrograph of pulmonary pyogranulomatous changes after a single intratracheal dose of bleomycin (0.075 U) in NMRI female mice. Interstitial chronic inflammation and intra-alveolar foamy macrophages are shown $(H \& E, \times 400$ magnification).

of lung were stained with hematoxylin and eosin (H \& E) or Masson's trichrome and examined by light microscopy.

Some lung sections showed multifocal foamy macrophages at three and four weeks after treatment with the single intratracheal dose of bleomycin. One case had histological changes involving the appearance of intra-alveolar and interstitial accumulation of lipid-laden, foamy macrophages (Fig. 1). There was also severe distortion of the pulmonary cytoarchitectural structure and multifocal fibrotic areas characterized by fibrous thickening

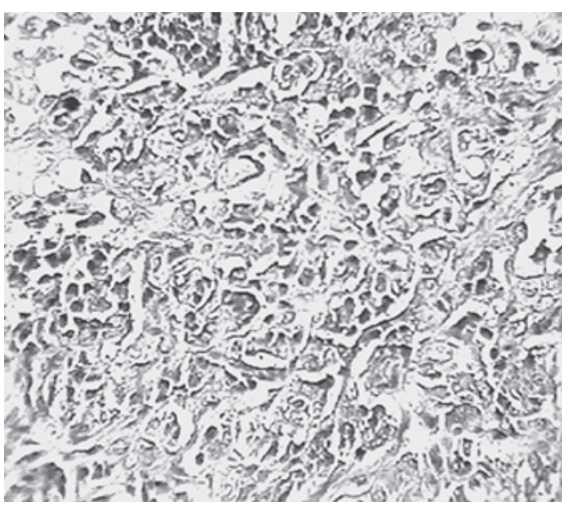

FIGURE 2 | Black and white photomicrograph of pulmonary fibrosis after a single intratracheal dose of bleomycin $(0.075 \mathrm{U})$ in NMRI female mice. Infiltration of inflammatory cells, increase in cellularity of alveolar septa, and fibrous thickening of alveolar walls with collagenous bands are shown (Masson's trichrome stain, $\times 400$ magnification).

of alveolar walls, interstitial inflammation with a pronounced neutrophilic increase in the alveolar septa, type II cell hyperplasia, and formation of fibrous bands and masses (Fig. 2).

Is this lesion what you expect from bleomycin-induced pulmonary damage? If not, how would you describe the lung sections with multifocal foamy macrophages? Is there another antineoplastic agent that induces these changes experimentally in lungs?

\section{What's your diagnosis?}

